\title{
Effect of Siwak and Bamboo Fibers on Tensile Properties of Self-Cure Acrylic Resin Used for Denture Applications
}

\author{
Jawad K Oleiwi*, Sihama I Salih and Hwazen S Fadhil \\ Department of Materials Engineering/University of Technology, Baghdad, Iraq
}

\begin{abstract}
The research investigated to determine enhancement in the tensile properties of self-cure acrylic resin reinforced with siwak fiber and bamboo fibers which were cut into 2, 6, and $12 \mathrm{~mm}$ lengths and used at three different concentrations of $\left(3,6\right.$, and $9 \mathrm{wt} \%$ ). The mixture of resin and fiber were cured at 2.5 bar and $55^{\circ} \mathrm{C}$ in a water bath for $30 \mathrm{~min}$. The cured resin specimen tested for tensile properties (tensile strength, young modulus, elongation percentage at break) following the specification of ASTM No. 638. The results illustrated that the tensile strength and young modulus tended to be improved with fiber length and concentration, the largest values of tensile strength and young modulus for specimens reinforced with bamboo fibers are (72.4 MPa and 5.208 GPa) while for specimens reinforced with siwak fibers are $(71 \mathrm{MPa}$ and $4.9 \mathrm{GPa})$ at optimum condition of weight fraction $(9 \%)$ and fiber length $(12 \mathrm{~mm})$ which was significantly higher than other formulations.
\end{abstract}

Keywords: Siwak; Bamboo; Tensile; Self cure; Acrylic resin; Weight fraction; Fiber length

\section{Introduction}

Acrylic resin is the most popular material used for denture base construction since 1937. It is better than other material in terms of aesthetics, simple technique at room temperature, and in-expensive cost. However, it has lower strength through dropping on a hard surface. The best way to enhance strength of acrylic resin is adding some type of fiber to it [1].

Teoman et al. [2] measured the effect of five types of fiber (glass, carbon, thin Kevlar, thick Kevlar, and polyethylene) reinforcement in woven form on the resistance to fracture of heat-polymerized denture base resin. The results show that the highest impact values obtained by reinforcing with polyethylene fibers, but there were no significant difference in transverse strength between specimens reinforced with polyethylene fibers and non-reinforced by fibers.

Pau-SuYen et al. [1] estimated enhancements in the mechanical properties of acrylic resin reinforced with different kinds of fiber (Polyester, Kevlar and glass) which utilized in three different length (2, 4 , and $6 \mathrm{~mm}$ ). The results illustrated that the impact strength improved with content and fiber length, especially when reinforcing by polyester fibers at weight fraction of ( $3 \mathrm{wt} . \%$ ) and length of $6 \mathrm{~mm}$, while there is no change in Bending strength make comparison with pure PMMA [1].

Tacir et al. [3] studied the influence reinforcement by glass fiber on flexural strength and fracture resistance of acrylic resin denture base, this study was indicated that the flexural strength of heat PMMA improved by reinforcing with glass fiber.

Doğan et al. [4] the researcher studied the effect of reinforcement denture base material with five aesthetic fibers at constant concentration of (3\%) by weight and three different length $(2,4,6 \mathrm{~mm})$. The result illustrated that the flexural strength, modulus of elasticity and max. load enhanced by nylon fibers of $(6 \mathrm{~mm})$ length, while there is no effect of incorporation different fibers in varying lengths on flexural property of the PMMA.

Khalaf [5] determined the effect of the addition of siwak micro powder on the Certain Mechanical Properties of Acrylic Resin. The results showed that the addition of (7\%) siwak powder to the Acrylic Resin revealed a significant decrease in compressive strength, impact strength and tensile strength.
Salih et al. [6,7] added nano-hydroxyapatite (nHA) particles and micro-zirconia $\left(\mathrm{ZrO}_{2}\right)$ particles in three volume fractions of $(1,2$ and $3) \%$ (PMMA) resin. Also glass fiber (E glass) and Kevlar fiber (49) in woven form added to PMMA at a fixed volume fraction of $5 \%$ and found that the magnitude of tensile properties enhanced with increasing the vol.\% of ( $\mathrm{nHA}$ and $\mathrm{ZrO}_{2}$ ) powders for all specimens while, elongation decreased with increasing volume fraction of (nHA and $\mathrm{ZrO}_{2}$ ) particles. And the composite (PMMA-ZrO ${ }_{2}$ ) show greater values for tensile strength and young modulus properties. While, the reinforcement by nHA particles had shown greater values for elongation for all groups' specimens. Also the results shown the maximum values for hybrid laminated composite materials for tensile strength and young modulus properties is observed in hybrid laminated composite materials (PMMA-ZrO ${ }_{2}$ ) and Kevlar fiber layer specimens. While the maximum value for elongation is obtained for first groups' specimens (PMMA resin reinforced by $\mathrm{nHA}$ particles).

Salih et al. [8] develop PMMA properties by addition of four kinds of nanoparticles, which are fly ash, fly dust, zirconia and aluminum in different ratios of volume fractions of (1\%,2\% and 3\%) to (PMMA) resin (castavaria) is the new fluid resin (pour type) as a matrix. The results show that the values of the hardness, flexural strength, Maximum shear stress and flexural modules increased and with the addition of Nano powders (fly ash, fly dust, zirconia, and aluminum) [8].

The aim of this research is studying the effect of natural fibers presented by siwak and bamboo fibers with different length and different weight fraction on tensile properties of the self-cure acrylic resin that used in dental applications.

*Corresponding author: Jawad K Oleiwi, Department of Materials Engineering, University of Technology, Baghdad, Iraq; E-mail: jawadkad@yahoo.com

Received August 03, 2017; Accepted August 21, 2017; Published August 31 2017

Citation: Oleiwi JK, Salih SI, Fadhil HS (2017) Effect of Siwak and Bamboo Fibers on Tensile Properties of Self-Cure Acrylic Resin Used for Denture Applications. J Material Sci Eng 6: 370. doi: 10.4172/2169-0022.1000370

Copyright: ( 2017 Oleiwi JK, et al. This is an open-access article distributed under the terms of the Creative Commons Attribution License, which permits unrestricted use, distribution, and reproduction in any medium, provided the original author and source are credited. 


\section{Materials and Methods}

\section{Material used}

In this research the Duracryl Plus self-curing base resin, manufactured by Spofa Dental Company. This type of material characterized by many properties compared with other kind of PMMA polymer, such as: softer feel, low molecular weight, color stable in the long run, minimized shrinkage, stable polymerization cycle with a perfect end result, the acrylic is long pourable and modelable for a long period of time [9]. Table 1 shows the properties of PMMA as obtain from the company. Figure 1 shows the methyl methacrylate powder and monomer that are used to fabricate composite specimens in this study.

Two types of fibers (bamboo fibers and siwak fibers) were used in this study as reinforcing materials with weight fraction of (3, 6 and 9\%) and length $(2,6$ and $12 \mathrm{~mm})$ it was added to PMMA. These types of fibers has a yellow color. Figures 2 and 3 show bamboo fibers and siwak fibers.

\section{Preparation methods of acrylic specimens}

Self-curing base resin is mixed in the volumetric ratio 3:1 (three parts of powder, 1 part of liquid). The mixing ratio is important because it is affect the acrylic resin cytotoxicity, setting dimensional changes and control the mixture workability.

\begin{tabular}{|l|c|}
\hline Property & Value \\
\hline Brinell hardness & Min. $120 \mathrm{Mpa}$ \\
\hline Bending strength & Min. $65.5 \mathrm{Mpa}$ \\
\hline Setting time & Max. $7 \mathrm{~min}$ \\
\hline Time of solubility & Max. $4 \mathrm{~min}$ \\
\hline Time required to prepare nontacky plastic mixture & $4-6 \mathrm{~min}$ \\
\hline Resistance to impact & Min. $0.40 \mathrm{~J} / \mathrm{cm}^{2}$ \\
\hline Absorbability & Max. $32 \mathrm{mg} / \mathrm{mm}^{3}$ \\
\hline Solubility & Max. $8 \mathrm{mg} / \mathrm{mm}^{3}$ \\
\hline
\end{tabular}

Table 1: Properties of PMMA.

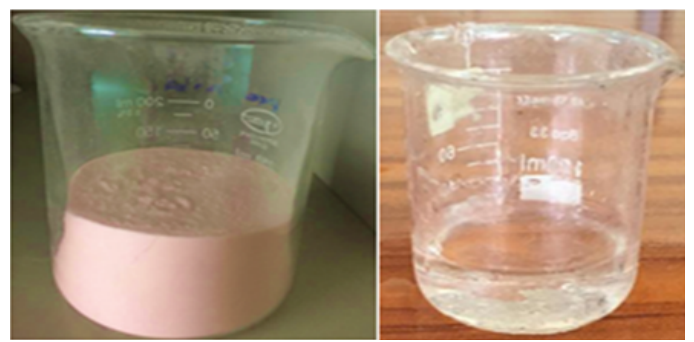

Figure 1: The powder PMMA and monomer MMA are used in this study.

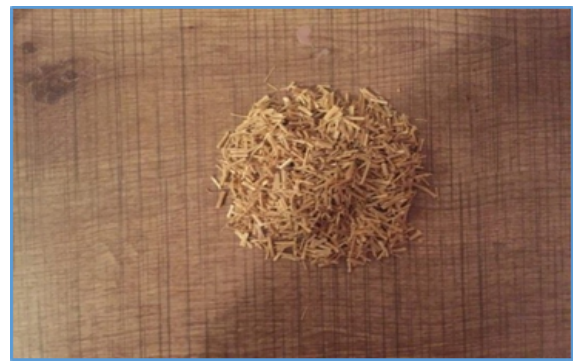

Figure 2: Sample of Bamboo Fibers.

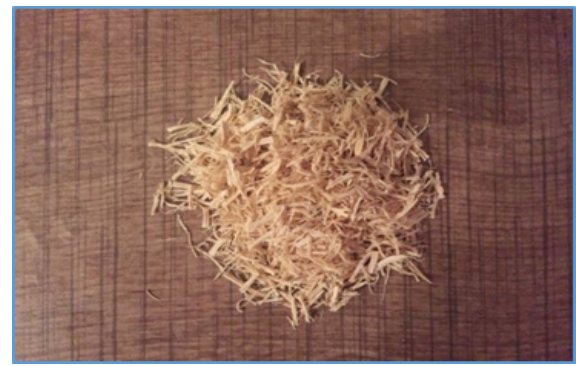

Figure 3: Sample of Siwak Fibers.

The mixture was mixed by using wood stick to prevent the chemical interaction at room temperature continuously by using hand in one direction for around 20 seconds and then poured into the metallic mold. The inner face of the mould was covered with a layer of smooth thermal nylon papers to ensure non adhesion of acrylic with the mould inner face and to obtain smooth lower surface with maximum time around $(4 \mathrm{~min})$ after pouring completion into the metallic mould the upper surface of the mould was covered with smooth thermal nylon and pressed by using metallic plate to obtain samples with smooth upper surface and prevent the vapor entry to acrylic resin during curing process inside Ivo met. The samples were finished with using special hand grinder to remove the cracks from the specimen's sides as a result of the specimen's adhesion with the metallic mould cavity sides.

The curing process of acrylic performed by placing the closed metallic mould inside the Ivo met at temperature equal to around $\left(55^{\circ} \mathrm{C}\right)$, and pressure equal to around $(2.5 \mathrm{bar})$, the closed mould remained inside Ivo met device for around $(30 \mathrm{~min})$ in order to complete polymerization process of acrylic specimens and to improve physical properties, the characteristic of this process is the residual monomer will be at minimum level and the polymerization process might be completed in short time.

\section{Preparation of composites specimens}

According to required selection ratio of weight fractions of the reinforcement materials weighing the amount of reinforced material (siwak fibers, Bamboo fibers) by using electronic balance with accuracy (0.0001) digits depending on total weight of the matrix material PMMA required for filling the mould cavities by using theory of rule of mixture. The fibers alkali treated with $5 \%(\mathrm{w} / \mathrm{v})$ alkali (sodium hydroxide) solution at $25^{\circ} \mathrm{C}$ for $24 \mathrm{~h}$, maintaining a fiber-to-liquor ratio of 1:30 (w/v). The fibers which alkali treated washed several times with distilled water to remove excess alkali sticking on their surface, then neutralized (PH-7) with distilled water containing a few drop of acetic acid and finally washed with distilled water, then the treated fibers were dried at room temperature for 5 days and finally kept in hot air oven at $\left(50-60^{\circ} \mathrm{C}\right)$ until dry. The liquid monomer (MMA) with one type of reinforcement fibers (Bamboo or siwak) should be mixed together at room temperature homogeneous and continuously, so, it must be sure of homogeneity of the mixture, before added powder to it to produce composite materials. The powder then added to the mixture and gradually mixed, then cured in same manner which indicated above.

The acrylic resin samples were de-molded to remove from the metallic mould cavities with very smooth upper and lower surface, followed by heat treatment at $55^{\circ} \mathrm{C}$ for $3 \mathrm{~h}$ to remove residual stresses as a result of de-molding of the specimens from the metallic mold cavity. 


\section{Tensile test}

Tensile tests were performed in this study to evaluate the tensile strength, modulus of elasticity, percentage of elongation of the cold cure composite materials. Tensile specimens and test is achieved according to ASTM (D-638) [10] at room temperature by utilizing the universal tensile instrument type (LARYEE) with capacity load (50 KN). The strain rate (speed of cross head) was $2 \mathrm{~mm} / \mathrm{min}$ and the tensile load was applied gradually until the fracture of the sample occurs. Figures 4 and 5 show the composite specimens before and after the test. Figure 6 shows the standard test specimen for tensile test.

\section{Stress-Strain curves}

The curves of (stress-strain) of pure PMMA, and the composite specimen reinforced with bamboo fibers, siwak fibers for length of $(2$ $\mathrm{mm}, 6 \mathrm{~mm}$ and $12 \mathrm{~mm}$ ) at different weight fraction of (3, 6 and $9 \mathrm{wt} . \%)$ are presented in Figures 7-13.

The stress-strain curves show linear behavior and then to nonlinear path. The linearity stage occurs due to the fibers deformation (bamboo and siwak). And the nonlinear stage is due to the matrix deformation

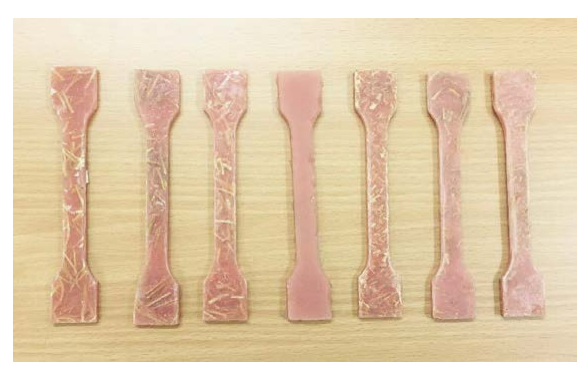

Figure 4: Specimens Before Test .



Figure 5: Specimens After Test.
[11]. Figures illustrate the strain increases with increasing in stress at different rates for different weight fractions and different fibers length.

\section{Results and Discussion}

\section{Tensile strength results}

Tensile strength values results discussed in Figures 14 and 15. It can be noted that the tensile strength increases slightly with increasing weight fraction of reinforcing fibers [12], the reasons behind such behavior is that the strengthening mechanism of reinforcing fibers in which, the amount of these fibers plays an important role impedes increasing the slipping of PMMA resin chains. It is worth noting the chains require high stress to bend in narrow space among the fibers. Also the interface bonding between the reinforcing fibers and matrix and has an essential part, so the results composite will demand high value of stress to break their interface bonding. The tensile strength increases with increasing weight percentage of fiber. If the fiber weight ratio decrease, load is not unequally transferred to fibers, which are not good bonded between matrix and fiber resulting reduces in tensile parameter [13]. Specimens with bamboo fibers reinforcement have relatively higher strength than the specimens with siwak fibers reinforcement. The difference in strength for these specimens is because of the difference in properties for bamboo and siwak fibers.

So, the greatest value of tensile strength for composite specimen reinforcing with bamboo fibers and siwak fibers at (9 wt.\%) weight fraction and $(12 \mathrm{~mm})$ fiber length reach $(72.4$ and $71 \mathrm{MPa})$, respectively.

\section{Young modulus results}

Young modulus values results discussed in Figures 16 and 17. It can be noted that the young modulus increases with increasing fiber length and weight fraction. So, the weight fraction of (9 wt.\%) and (12 mm) fiber length represent the greatest value for the modulus of elasticity for PMMA reinforced with bamboo or siwak fibers. The reason behind increasing young's modulus value with increasing content of fibers may be due to the fact that fibers have good stiffness than resin because they have young modulus higher than matrix and that leads to improving the stiffness of the composite. Also as the fiber weight fraction increased, there is possibility of fiber-matrix interaction leads to an increase in efficiency of stress transfer from the matrix phase to the fiber phase.

From these figures, also it can be seen that bamboo fibers improve young modulus more than siwak fibers, this is due to the fact that mechanical properties of bamboo fibers higher than siwak fibers $[14,15]$.

PMMA resin specimen had (1.7 GPa), but when adding bamboo reinforcing fibers, the young modulus reaches to $(5.2 \mathrm{GPa})$ at $(9 \mathrm{wt} . \%)$

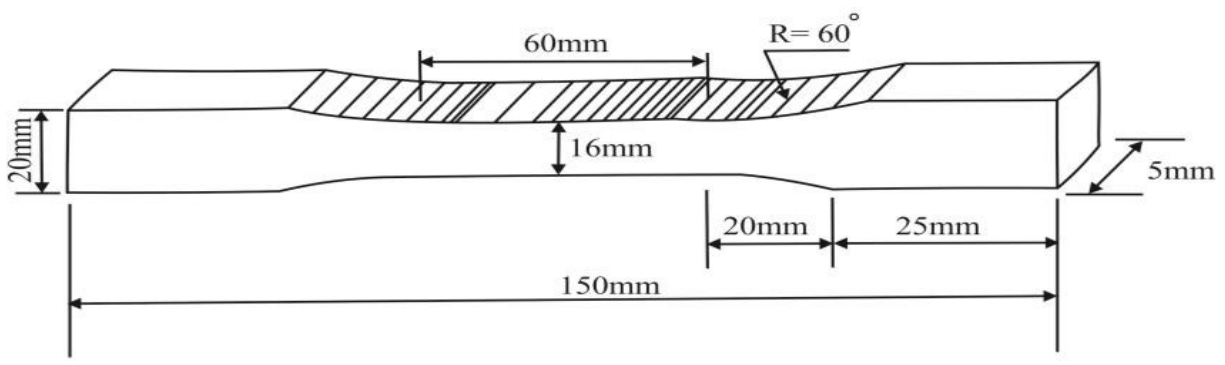

Figure 6: Standard tensile test specimen. 
Citation: Oleiwi JK, Salih SI, Fadhil HS (2017) Effect of Siwak and Bamboo Fibers on Tensile Properties of Self-Cure Acrylic Resin Used for Denture Applications. J Material Sci Eng 6: 370. doi: 10.4172/2169-0022.1000370



Figure 7: Stress-Strain Curve for Pure (PMMA) Specimen



Figure 8: Stress-Strain curve for composite specimen with (3wt.\%) bamboo fibers for (2, 6 and $12 \mathrm{~mm})$ fiber length.

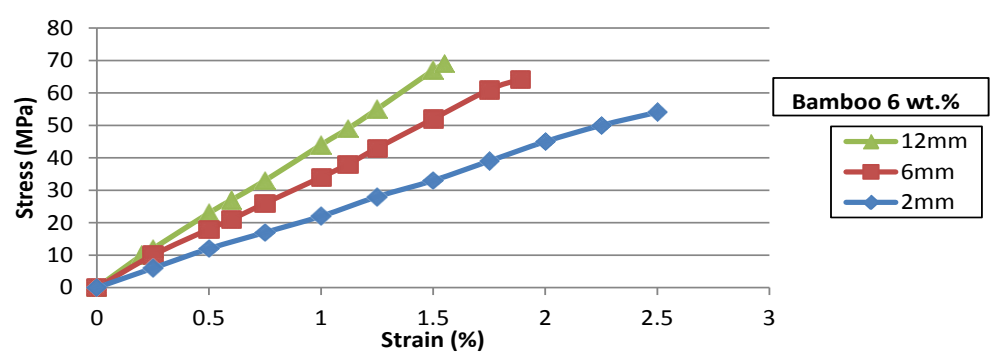

Figure 9: Stress-Strain curve for composite specimen with (6wt.\%) bamboo fibers for (2, 6 and $12 \mathrm{~mm})$ fiber length.

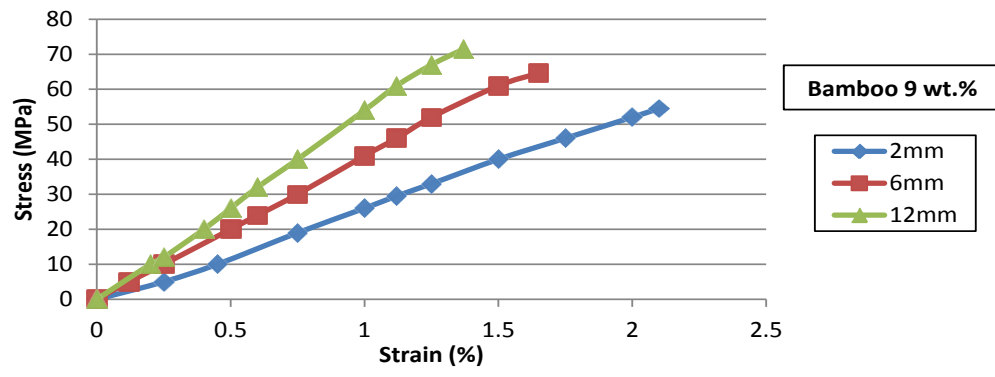

Figure 10: Stress-Strain curve for composite specimen with (9 wt. \%) bamboo fibers for (2, 6 and $12 \mathrm{~mm})$ fiber length.

and (12 $\mathrm{mm})$, While with siwak reinforcing fibers, the value reaches to $(4.8 \mathrm{GPa})$ in this study for the same conditions.

\section{Elongation percentage at break}

The percentage of elongation determined at fracture, and fibers content reinforcement (bamboo and siwak) of $(2 \mathrm{~mm}, 6 \mathrm{~mm}$ and $12 \mathrm{~mm}$ ) length, which was added to the Poly Methyl Methacrylate resin is shown in Figures 18 and 19. Increasing the weight fraction of (bamboo or siwak) reinforcing fibers leads to reduce the percentage of elongation of composite specimens. This is because the presence of reinforcement imparts the stiffening effect within the resin and thus imposes a mechanical restraint on the composite. Also decreases of elongation percentage depend on the interface that is between fiber and matrix $[16,17]$.

It can be seen from the figures that, the lower value was found with specimens at $12 \mathrm{~mm}$ length and (9\%) weight fraction for both bamboo 


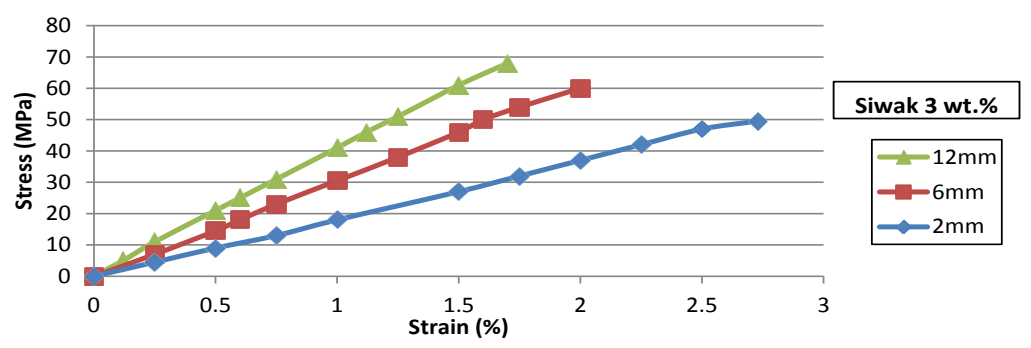

Figure 11: Stress-Strain curve for composite specimen with (3 wt.\%) siwak fibers for (2, 6 and $12 \mathrm{~mm})$ fiber length.

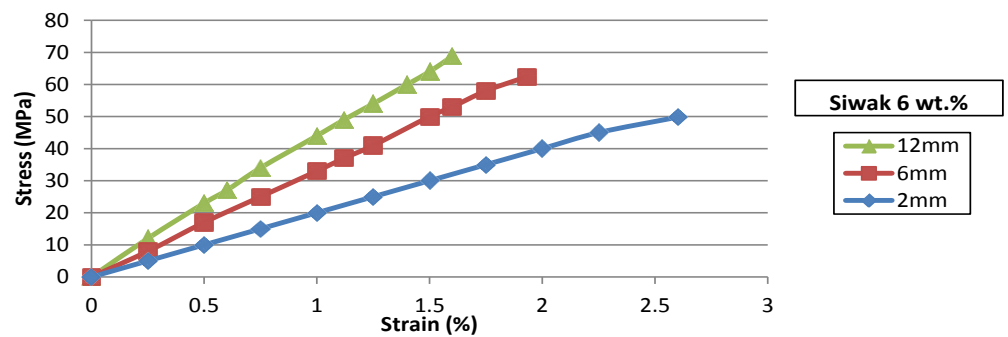

Figure 12: Stress-Strain curve for composite specimen with (6 wt.\%) siwak fibers for (2,6 and12 mm) fiber length.



Figure 13: Stress-Strain curve For composite specimen with ( $9 \mathrm{wt} . \%)$ siwak fibers for $(2,6$ and12 $\mathrm{mm})$ fiber length.

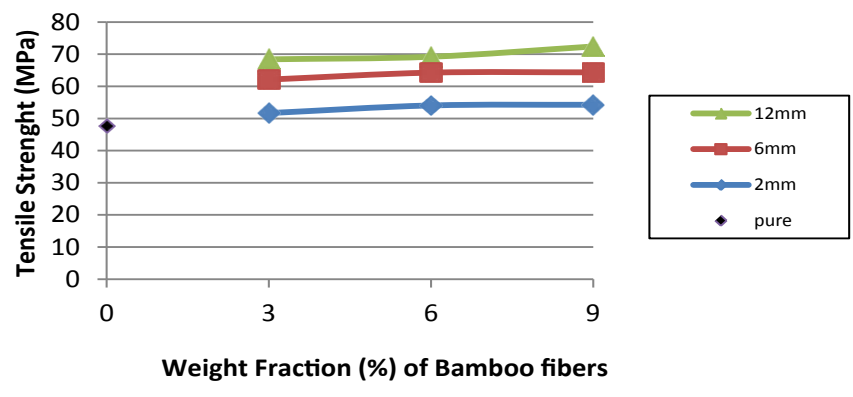

Figure 14: Tensile strength and weight fraction of bamboo fibers at different fiber length.

and siwak fibers. The percent of elongation at break for composite specimen with bamboo fibers reach $(1.37 \%)$, while for composite specimen with siwak fibers reach (1.45\%).

Specimens of siwak fibers have percentage of elongation more than with bamboo fibers, due to bamboo fibers have good bonding, that resist extensions more than siwak fibers and also to properties of bamboo and siwak fibers.

\section{Conclusions}

- Pure PMMA had the lower value of tensile strength and

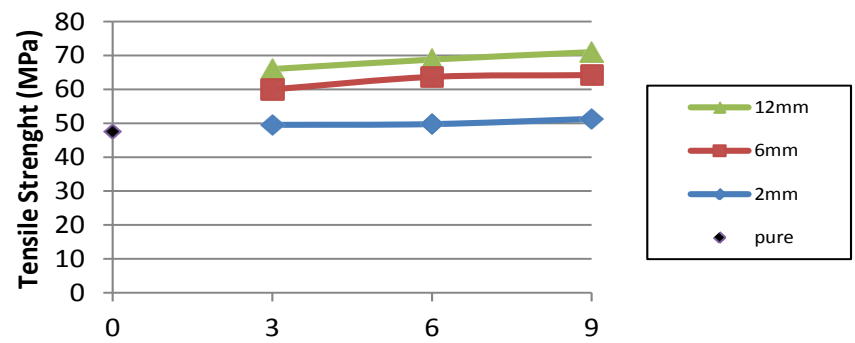

Weight Fraction (\%) of Siwak fibers

Figure 15: Tensile strength and weight fraction of siwak fibers at different fiber length.

modulus of elasticity which equal (47.6 MPa and 1.7 GPa), respectively.

- Tensile strength, young modulus increase with the increase in the weight fraction and fiber length of the reinforcing fibers (bamboo and siwak) in PMMA resin.

- Elongation percentage at break decrease with increasing both weight fraction and fiber length for both types of fibers.

- The largest values of tensile strength and young modulus for specimens reinforced with bamboo fibers are (72.4 MPa and 
Citation: Oleiwi JK, Salih SI, Fadhil HS (2017) Effect of Siwak and Bamboo Fibers on Tensile Properties of Self-Cure Acrylic Resin Used for Denture Applications. J Material Sci Eng 6: 370. doi: 10.4172/2169-0022.1000370

Page 6 of 6

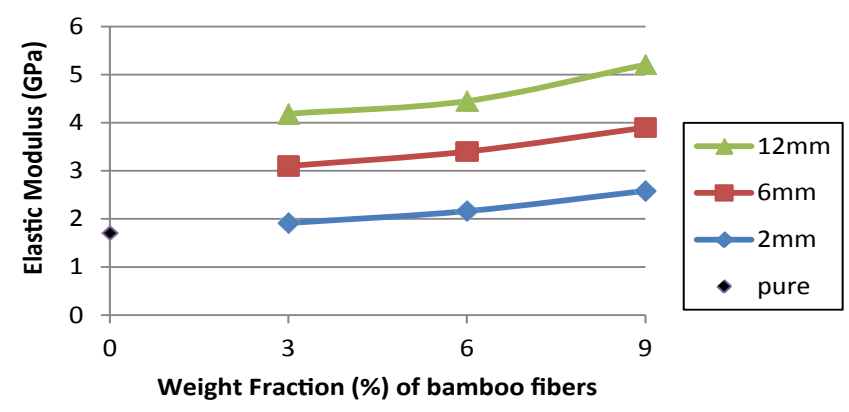

Figure 16: Young's modulus and weight fraction of bamboo fibers at different fiber length.

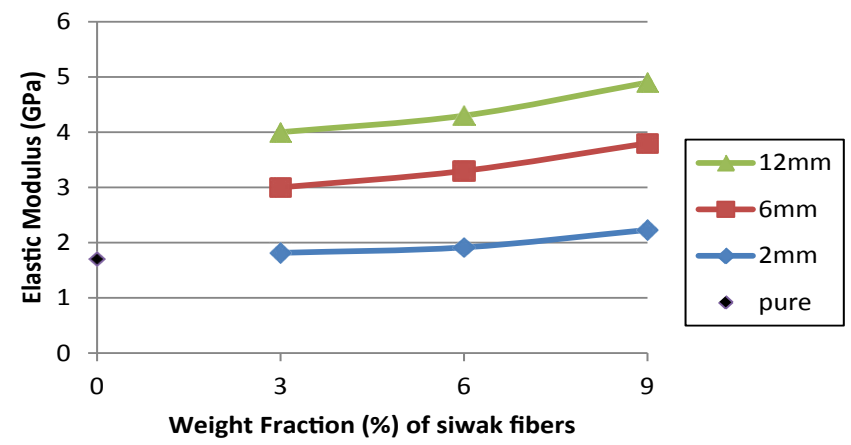

Figure 17: Young's modulus and weight fraction of siwak fibers at different fiber length.

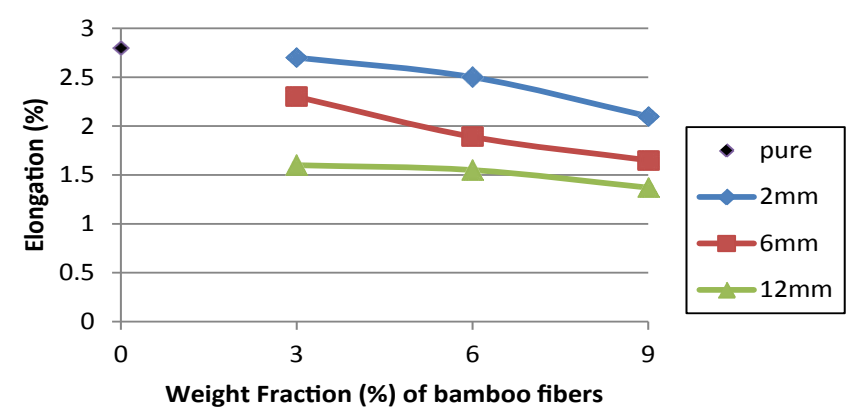

Figure 18: Elongation percentage at break with weight fraction of bamboo fibers.

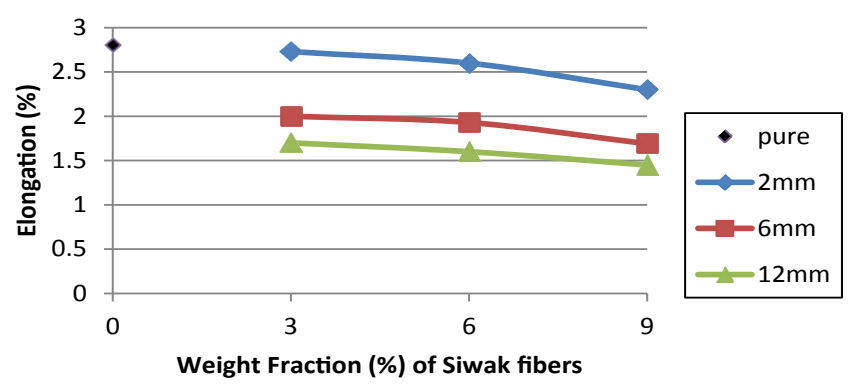

Figure 19: Elongation percentage at break with weight fraction of siwak fibers.

$5.208 \mathrm{GPa}$ ) while for specimens reinforced with siwak fibers are (71 MPa and $4.9 \mathrm{GPa}$ ) at optimum condition of weight fraction (9\%) and fiber length (12 mm).

\section{References}

1. Chen SY, Liang WM, Yen PS (2001) Reinforcement of acrylic denture base resin by incorporation of various fibers. Journal of Biomedical Materials Research Part A 58: 203-208.

2. Uzun G, Hersek N, Tincer T (1999) Effect of five woven fiber reinforcements on the impact and transverse strength of a denture base resin. The Journal of prosthetic dentistry 81: 616-620.

3. Tacir IH, Kama JD, Zortuk M, Eskimez S (2006) Flexural properties of glass fibre reinforced acrylic resin polymers. Australian Dental Journal 51: 52-56.

4. Doğan OM, Bolayır G, Keskin S, Doğan A, Bek B (2008) The evaluation of some flexural properties of a denture base resin reinforced with various aesthetic fibers. Journal of Materials Science: Materials in Medicine 19: 2343-2349.

5. Khalaf HAR (2013) Effect of Siwak on Certain Mechanical Properties of Acrylic Resin. Journal of Oral and Dental Research 1: 40-45.

6. Salih APDSI, Oleiwi APDJK, Hamad LQA (2014) Studying the Tensile Properties and Morphology Test for the Self Cured PMMA Resin of Prosthetic Complete Denture.

7. Salih SI, Oleiwi JK, Hamad QA (2015) Numerically and Theoretically Studying of the Upper Composite Complete Prosthetic Denture. Eng \& Tech Journal Part (A) 33: 1023-1037.

8. Salih SI, Oleiwi JW, Alaa MT (2016) Investigation of Hardness and Flexural Properties of PMMA Nano Composites and PMMA Hybrids Nano Composites Reinforced by Different Nano Particles Materials used in Dental Applications. Engineering and Technology Journal 34.

9. O'Brien WJ (2002) Dental materials and their selection. (3rdedn), Chicago: Quintessence Int, pp: 216-20.

10. Standard ASTM (2009) Standard test method for tensile properties of plastics, D 638-08. Annual book of ASTM standards, p: 8.

11. Faizal MA, Beng YK, Dalimin MN (2006) Tensile property of hand lay-up plain-weave woven e glass/polyester composite: curing pressure and ply arrangement effect. Borneo Sci 19: 27-34.

12. Hashim AM (2016) Tensile and Fracture Properties of Coir Fiber Green Composites Bone Plate Fixation. ZANCO Journal of Pure and Applied Sciences 28.

13. Venkateshwaran N, ElayaPerumal A, Jagatheeshwaran MS (2011) Effect of fiber length and fiber content on mechanical properties of banana fiber/epoxy composite. Journal of Reinforced Plastics and Composites 30: 1621-1627.

14. Gupta SK (2014) A study on mechanical behavior of bamboo fiber based polymer composites (Doctoral dissertation).

15. Moawed EA (2013) Effect of heating processes on Salvadora persica (Miswak) and its application for removal and determination of aniline blue from wastewater. Journal of Taibah University for Science 7: 26-34.

16. Sreekanth MS, Bambole VA, Mhaske ST, Mahanwar PA (2009) Effect of concentration of mica on properties of polyester thermoplastic elastomer composites. Journal of Minerals and Materials Characterization and Engineering 8: 271.

17. Sawalha S, Mosleh A, Manasrah A (2007) Tensile Properties of Extruded Short Glass Fiber/Low Density Polyethlene Composites. Iranian Polymer Journal 16: 719-726. 